\title{
Human-Related Emotional Stimuli Can Cause a Hippocampal and Thalamic Over-Response in People with Unstable Personalities
}

\author{
Yuko Mizuno-Matsumoto ${ }^{1^{*}}$, Takuto Hayashi ${ }^{2}$, Eika Okamoto $^{1}$, Daisuke Miwa ${ }^{1}$, \\ Tetsuya Asakawa ${ }^{3}$, Ayumi Muramatsu ${ }^{1}$, Makoto Kato ${ }^{4}$, Tsutomu Murata ${ }^{4}$ \\ ${ }^{1}$ Graduate School of Applied Informatics, University of Hyogo, Hyogo, Japan \\ ${ }^{2}$ Department of Medical Engineering, Aino University, Osaka, Japan \\ ${ }^{3}$ Faculty of Physical Education, Osaka University of Health and Sport Sciences, Osaka, Japan \\ ${ }^{4}$ Center for Information and Neural Networks, Advanced ICT Research Institute, \\ National Institute of Information and Communications Technology (NICT), Hyogo, Japan \\ Email: ${ }^{*}$ yuko@ai.u-hyogo.ac.jp
}

Received August 26, 2013; revised September 28, 2013; accepted October 8, 2013

Copyright (C) 2013 Yuko Mizuno-Matsumoto et al. This is an open access article distributed under the Creative Commons Attribution License, which permits unrestricted use, distribution, and reproduction in any medium, provided the original work is properly cited.

\begin{abstract}
Hippocampus is crucial for the formation of emotional memory. We found the relationship between hippocampal responses to emotional stimuli and the mental stabilities of people in our preliminary study. In this study, we have also evaluated how the emotional stimuli would affect amygdala and thalamus in the brain, and how the personality stabilities could relate to the responses in the brain using functional magnetic resonance imaging (fMRI). We evaluated the subjects' personality features with the Yatabe-Guilford Personality Test (Y-G test) and psychosomatic symptoms with the Cornell Medical Index (CMI). The subjects were categorized into the mentally stable group and the mentally unstable group according to the total scores of the Y-G test and the CMI. The brain functional responses under emotional stimuli were measured using fMRI. The region of interest (ROI) analysis was performed to abstract significant changes in order to compare responses among the different emotional stimuli. We conducted the regression analysis to abstract the relationship between the mean \% signal change from fMRI and the personality stability. The fMRI results showed that the hippocampus, thalamus, and right amygdala activities under the human relationship stimuli increased with ascending value of mental instability. Our findings suggest that the memory process in the hippocampus and the threat alarm system in the thalamus under the human-related stimuli crucially influence the emotional reaction of mentally unstable people. These processes in the brain would affect the event that stresses on human relationships that often cause people to suffer from mental disorders.
\end{abstract}

Keywords: fMRI; Emotion; Personality; Hippocampus; Thalamus; Amygdala

\section{Introduction}

Mental and behavioral disturbances in adults who have been labeled social misfits are an increasingly serious social issue related to the growing numbers of people who suffer from depression. The lifetime prevalence of depression is $3 \%$ to $16 \%$ of people, and, in Japan, 433,000 people and 1,041,000 people are reported to have suffered from mood disorders in 1996 and 2008, respectively [1]. The Japanese Ministry of Health, Labour and Welfare in a Comprehensive Survey of Living

\footnotetext{
${ }^{*}$ Corresponding author.
}

Conditions, has reported that more than half of the people in Japan have had some distress and feel stress in their lives [2]. The Ministry in a Survey of Sate Employees' Health also has reported that the top cause of worry and stress for people centered on their human relationships at work [3]. Information relevant to unpleasant stress is thought to be processed in the prefrontal area, the hippocampus, and the amygdala [4]. In processing, the amygdala regulates the emotional stimuli that refer to memory in the hippocampus. The brain function elicits an emotional reaction mentally according to the particular memory. In addition, the emotional stimuli simultaneously 
evoke physical reactions such as changes in blood pressure, pulse waves, and body temperature via an autonomic nervous system. Therefore, when personality stability is assessed, both emotional and physical stabilities need to be evaluated. Moreover, the brain reaction formed from the emotional stimuli would be different with different types of mental stability.

We found the relationship between hippocampal responses to emotional stimuli and the mental stabilities of people in our preliminary study. In this study, we have also evaluated how the emotional stimuli would affect amygdala and thalamus in the brain, and how the personality stabilities could relate to the responses in the brain using functional magnetic resonance imaging (fMRI).

The objective of this research is to assess the relationship between functional responses in the brain to emotional stimuli and the personality stabilities of people using fMRI.

\section{Methods}

\subsection{Subject}

The subjects were 33 healthy young adults (18 males, 15 females; 21 - 38 years old, mean 24.1 years old, S.D. 3.5). They had no history of neurological or psychiatric illness. The Ethical Committee of the University of Hyogo approved this investigation and informed consent was obtained according to the Declaration of Helsinki.

\subsection{Psychological Tests}

We used two kinds of questionnaires to evaluate personality stability in the mental and psychosomatic aspects of the subjects.

1) The Yatabe-Guilford Personality Test (Y-G Test)

Using the $\mathrm{Y}-\mathrm{G}$ test, personality features including the emotional stability of the subjects were assessed [5]. People were classified into 5 personality types according to 12 personality factors in the $\mathrm{Y}-\mathrm{G}$ test. The five personality types are type $\mathrm{C}$ (the calm type), type $\mathrm{D}$ (the director type), type A (the average type), type $\mathrm{E}$ (the eccentric type), and type $\mathrm{B}$ (the black list type). We gave the score, $0,1,2,3$, and 4 to type $\mathrm{C}, \mathrm{D}, \mathrm{A}, \mathrm{E}$, and $\mathrm{B}$, respectively. The larger the score, the lower the personality stability of the subject.

2) The Cornell Medical Index (CMI)

Using the CMI, subjects were assessed as to the presence of physically unstable and neurotic states, and they were categorized into type I (healthy), type II (psychosomatically slightly ill), type III (psychosomatically ill), and type IV (psychosomatically severely ill, neurotic) [6]. We gave the scores, $0,1,2$, and 3 to types I, II, III, and IV, respectively. The larger the score, the lower the psychosomatic stability of the subject.

3) Categorizing the Subjects
The personality stabilities of the subjects were evaluated according to the total scores of the Y-G test and the CMI. Zero and 7 scores indicate the most stable and the most unstable in personality stability, respectively. The subjects were also divided into mentally stable or unstable groups according to the total scores of the Y-G test and the CMI. When the total score of the Y-G test and the CMI is zero to two and three to seven, the subject was categorized into the stable and unstable groups, respectively. In other words, the subjects with type $C$ in the Y-G test and type I, II or III in the CMI, with type D in the Y-G test and type I or II in the CMI, and with type A in the Y-G test and type I in the CMI were categorized into the stable group. The subjects with type $\mathrm{C}$ in the Y-G test and type IV in the CMI, type D in the Y-G test and type III and IV in the CMI, type A in the Y-G test and type II, III, and IV in the CMI and type E or B in the $\mathrm{Y}-\mathrm{G}$ test were categorized into the unstable group.

The mean ages between the stable and unstable groups were compared using a Student's t-test, and the male-tofemale ratios between the two groups were compared using Fisher's $\chi^{2}$ test.

\section{3. fMRI Experiments}

The fMRIs were measured in the subjects under emotional stimuli. The subjects were presented with calm and relaxing stimuli (relaxed stimuli), cheerful and pleasant stimuli (pleasant stimuli), unpleasant human relationships stimuli (human stimuli), and general unpleasant stimuli (unpleasant stimuli). In all the tasks, the visualauditory stimuli were adopted. For the visual-auditory stimuli, natural scenes and sound effects were presented in the relaxed stimuli, amusing images and up-tempo sounds in the pleasant stimuli, human-related stressful images and sound effects in the human stimuli, and scenes from horror movies in the unpleasant stimuli were presented to the subjects. After a 2 dummy scan at the beginning of the trial, 4 blocks were conducted. The trail order was counterbalanced in a run and each emotional stimulus order was randomized in an experiment. In 1 block, we randomly presented five 4-second visual-auditory stimuli consisting of the same kind of task for 20 seconds following a cross-hair image for 20 seconds. We conducted 6 trials and presented 30 stimuli in each task in total.

Structural and functional imaging data were acquired using a 3-T whole body scanner (Siemens MAGNETOM Trio, Germany) at Advanced ICT Research Institute, NICT, Kobe, Japan. Functional $\mathrm{T} 2{ }^{*}$-weighted images were acquired using a gradient echo-planar imaging (GRE-EPI) sequence. Forty consecutive axial slices (thickness $3 \mathrm{~mm}$, no gap) covering the entire cortex and cerebellum were acquired. Imaging data were analyzed using Statistical Parametric Mapping software (SPM5, 
Wellcome Department of Cognitive Neurology, London, UK). Preprocessing included slice time correction, motion correction (using a six-parameter, rigid body transformation algorithm by SPM5), normalization to the Montreal Neurological Institute (MNI) template (resampling at 3-mm isotropic resolution), and spatial smoothing (at a 6-mm isotropic Gaussian kernel). Statistical analysis relied on a general linear model. Analyses were performed for each individual and for the group. For group analyses, voxel-wise paired $t$ tests were performed using FWE (Familywise Error)-corrected thresholds combined with a cluster-size threshold of 5 voxels. We evaluated the whole brain and the ROI to contrast the brain areas. For each subject, a composite image contrasting the mean of the four emotion (relaxed, pleasant, human, and unpleasant) blocks with the mean of all of the neutral blocks was made for twelve anatomical ROIs. The twelve images from all 33 subjects were then averaged to create an overall group image and were used to locate the maximum positive correlation peak for the anatomical ROIs. We conducted the simple linear regression analysis to abstract the relationship between the mean \% signal change from fMRI and the personality stability.

\section{Results}

Table 1 shows the number, mean age, and male-to-female ratio in the stable and unstable groups. There was no significant difference in mean age between the two groups ( $t$-test), and there was no significant difference in the male-to-female ratio between the two groups (Fisher's $\chi^{2}$ test).

Table 2 shows the number of the subjects and the total score of the Y-G test and the CMI. White and gray columns show stable and unstable groups, respectively. As a consequence, 18 (total score $0-2 \mathrm{pt}$ ) and 15 subjects (total score 3 - $7 \mathrm{pt}$ ) were categorized into stable and unstable groups, respectively.

Table 3 shows the regions significantly activated during emotional stimuli in the stable and unstable groups ( $p$ $<0.01$ ). There were no significant activities in any region of both stable and unstable groups under relaxed stimuli. The left and right hippocampus of both groups were significantly activated in the pleasant, human, and unpleasant stimuli. The left and right amygdala of both groups

Table 1. Subjects information.

\begin{tabular}{cccc}
\hline & Stable group & Unstable group & $p$-value \\
\hline $\mathrm{N}$ & 18 & 15 & \\
Age & $25.1 \pm 4.2$ & $23.0 \pm 2.2$ & 0.114 \\
Sex (Male:Female) & $12: 6$ & $6: 9$ & 0.126 \\
\hline
\end{tabular}

Table 2. The number of the subjects and the total score of the Y-G test and CMI. Eighteen (score 0 - 2 pt) and fifteen subjects (score 3 - 7 pt) were categorized into stable and unstable groups, respectively.

\begin{tabular}{cccccc}
\hline \multicolumn{5}{c}{ CMI (score) } \\
\hline & I (0 pt) & II (1 pt) & III (2 pt) & IV (3 pt) \\
\hline & C (0 pt) & $6(0)$ & $1(1)$ & $0(2)$ & $0(3)$ \\
Y-G & D (1 pt) & $8(1)$ & $3(2)$ & $0(3)$ & $0(4)$ \\
$\begin{array}{c}\text { test } \\
\text { score })\end{array}$ & A (2 pt) & $0(2)$ & $2(3)$ & $3(4)$ & $0(5)$ \\
& E (3 pt) & $0(3)$ & $1(4)$ & $2(5)$ & $2(6)$ \\
& B (4 pt) & $0(4)$ & $0(5)$ & $5(6)$ & $0(7)$ \\
\hline
\end{tabular}

$\square$ stable group; $\square$ unstable group.

Table 3. Regions activated during emotional stimuli in stable and unstable groups. At least 5 voxels; $p<0.01$, FWEcorrected; L: Left, R: Right. MNI coordinates and T show at the maximum positive correlation peak.

\begin{tabular}{|c|c|c|c|c|c|}
\hline ROI definition & $\mathrm{L} / \mathrm{R}$ & $\begin{array}{l}\text { MNI coordinates } \\
{[\mathrm{mm}]}\end{array}$ & $\mathrm{T}$ & $\begin{array}{c}\text { Cluster } \\
\text { size }\end{array}$ & $\begin{array}{c}\text { Total } \\
\text { cluster } \\
\text { size }\end{array}$ \\
\hline \multicolumn{6}{|l|}{ Stable group } \\
\hline \multicolumn{6}{|l|}{ Pleasant } \\
\hline \multirow[t]{2}{*}{ Frontal_Inf_Oper } & $\mathrm{L}$ & $(-33,13,27)$ & 7.49 & 6 & 78 \\
\hline & $\mathrm{R}$ & $(42,13,27)$ & 8.84 & 72 & \\
\hline \multirow[t]{2}{*}{ Hippocampus } & $\mathrm{L}$ & $(-21,-24,-6)$ & 12.08 & 93 & 159 \\
\hline & $\mathrm{R}$ & $(24,-24,-6)$ & 9.54 & 66 & \\
\hline \multirow[t]{2}{*}{ Amygdala } & $\mathrm{L}$ & $(-24,-6,-10)$ & 7.47 & 53 & 94 \\
\hline & $\mathrm{R}$ & $(24,-1,-20)$ & 7.18 & 41 & \\
\hline \multirow[t]{2}{*}{ Thalamus } & $\mathrm{L}$ & $(-18,-26,-1)$ & 11.4 & 17 & 35 \\
\hline & $\mathrm{R}$ & $(21,-26,-1)$ & 11.66 & 18 & \\
\hline \multicolumn{6}{|l|}{ Human } \\
\hline \multirow[t]{2}{*}{ Frontal_Inf_Oper } & $\mathrm{L}$ & $(-33,10,24)$ & 6.02 & 8 & 97 \\
\hline & $\mathrm{R}$ & $(42,13,27)$ & 11.57 & 89 & \\
\hline SupraMarginal & $\mathrm{L}$ & $(-53,-37,24)$ & 6.48 & 6 & 6 \\
\hline \multirow[t]{2}{*}{ Hippocampus } & $\mathrm{L}$ & $(-21,-24,-6)$ & 13.23 & 117 & 225 \\
\hline & $\mathrm{R}$ & $(33,-24,-6)$ & 12.93 & 108 & \\
\hline \multirow[t]{2}{*}{ Amygdala } & $\mathrm{L}$ & $(-27,-4,-23)$ & 8.07 & 48 & 102 \\
\hline & $\mathrm{R}$ & $(24,-4,-17)$ & 10.48 & 54 & \\
\hline \multirow[t]{2}{*}{ Thalamus } & $\mathrm{L}$ & $(-18,-26,-1)$ & 11.79 & 36 & 61 \\
\hline & $\mathrm{R}$ & $(21,-26,-1)$ & 12.94 & 25 & \\
\hline Angular & $\mathrm{R}$ & $(30,-65,45)$ & 7.02 & 9 & 9 \\
\hline \multicolumn{6}{|l|}{ Unpleasant } \\
\hline Frontal_Inf_Oper & $\mathrm{R}$ & $(42,13,27)$ & 12.76 & 77 & 77 \\
\hline
\end{tabular}




\section{Continued}

\begin{tabular}{cccccc}
\hline Hippocampus & L & $(-15,-27,-6)$ & 14 & 90 & 145 \\
& R & $(33,-24,-6)$ & 10.86 & 55 & \\
Amygdala & L & $(-24,-1,-23)$ & 7.89 & 42 & 62 \\
& R & $(18,-1,-15)$ & 6.35 & 20 & \\
Thalamus & L & $\begin{array}{c}(-18,-26,-1) / \\
(-12,-11,6)\end{array}$ & $12.06 / 6.44$ & $20 / 20$ & 56 \\
& R & $(21,-26,-1)$ & 11.26 & 16 & \\
Angular & $\mathrm{R}$ & $(30,-65,45)$ & 8.83 & 15 & 15
\end{tabular}

Unstable group

Pleasant

\begin{tabular}{|c|c|c|c|c|c|}
\hline \multirow[t]{2}{*}{ Frontal_Inf_Oper } & $\mathrm{L}$ & $\begin{array}{c}(-39,13,27) / \\
(-42,13,19)\end{array}$ & $7.71 / 6.90$ & $7 / 7$ & 48 \\
\hline & $\mathrm{R}$ & $(45,16,27)$ & 10.17 & 34 & \\
\hline \multirow[t]{2}{*}{ Hippocampus } & $\mathrm{L}$ & $(-21,-27,-6)$ & 11.03 & 87 & 121 \\
\hline & $\mathrm{R}$ & $\begin{array}{l}(30,-24,-6) / \\
(33,-12,-15)\end{array}$ & 15.6 & 34 & \\
\hline \multirow[t]{2}{*}{ Amygdala } & $\mathrm{L}$ & $(-27,-4,-20)$ & 8.27 & 17 & 22 \\
\hline & $\mathrm{R}$ & $(30,-1,-20)$ & 5.78 & 5 & \\
\hline \multirow[t]{2}{*}{ Thalamus } & $\mathrm{L}$ & $\begin{array}{c}(-18,-26,-1) / \\
(-9,-8,6)\end{array}$ & $11.80 / 8.87$ & $16 / 22$ & 77 \\
\hline & $\mathrm{R}$ & $\begin{array}{c}(9,-11,3) / \\
(21,-26,-1)\end{array}$ & $8.74 / 12.15$ & $22 / 17$ & \\
\hline \multicolumn{6}{|l|}{ Human } \\
\hline \multirow[t]{2}{*}{ Frontal_Inf_Oper } & $\mathrm{L}$ & $(-36,13,27)$ & 7.57 & 8 & 38 \\
\hline & $\mathrm{R}$ & $(45,16,27)$ & 9.59 & 30 & \\
\hline \multirow[t]{2}{*}{ Hippocampus } & $\mathrm{L}$ & $(-21,-29,-4)$ & 12.99 & 80 & 159 \\
\hline & $\mathrm{R}$ & $(21,-7,-17)$ & 13.76 & 79 & \\
\hline \multirow[t]{2}{*}{ Amygdala } & $\mathrm{L}$ & $(-24,-4,-17)$ & 7.31 & 18 & 45 \\
\hline & $\mathrm{R}$ & $(30,-4,-17)$ & 12.36 & 27 & \\
\hline \multirow[t]{2}{*}{ Thalamus } & $\mathrm{L}$ & $\begin{array}{c}(-6,-12,-2) / \\
(-21,-26,1)\end{array}$ & $7.68 / 10.63$ & $18 / 17$ & 72 \\
\hline & $\mathrm{R}$ & $\begin{array}{c}(9,-11,3) / \\
(21,-26,-1)\end{array}$ & $8.67 / 10.79$ & $29 / 8$ & \\
\hline
\end{tabular}

Unpleasant

\begin{tabular}{cccccc} 
Frontal_Inf_Oper & L & $(-36,13,27)$ & 7.89 & 11 & 49 \\
& R & $(48,16,30)$ & 12.02 & 38 & \\
SupraMarginal & L & $(-62,-22,34)$ & 12.04 & 33 & 33 \\
Hippocampus & L & $(-21,-27,-6)$ & 16.9 & 74 & 158 \\
& R & $(27,-7,-15)$ & 13.33 & 84 & \\
Amygdala & L & $(-24,-9,-12)$ & 9.3 & 30 & 73 \\
& R & $(30,-6,-12)$ & 10.36 & 43 & \\
Thalamus & L & $(-18,-29,1)$ & 14.24 & 77 & 154 \\
& R & $(21,-23,-1)$ & 12.45 & 77 & \\
\hline
\end{tabular}

were significantly activated in the pleasant, human, and unpleasant stimuli. The left and right thalamus of both groups were significantly activated in the pleasant, human, and unpleasant stimuli.

Figure 1 shows the amygdala, hippocampus, and thalamus activities of the stable and unstable groups in relaxed, pleasant, human, and unpleasant stimuli in the coronal section. The amygdala and hippocampus of the stable group under pleasant stimuli and of both groups under human and unpleasant stimuli are activated.

Figures 2-4 show the relationship between mean \% signal change and personality stability in the amygdala, hippocampus, and thalamus by regression analysis, respectively. The left activity is shown with a triangle and a dashed line, and right activity is shown with a circle and a solid line. Only the significant and tended regression lines are shown in the Figures. The abscissa shows the degree of personality stability, and the ordinate shows the value of the mean \% signal change.

Figure 2 shows that the brain activities in the right amygdala under pleasant and human stimuli tended to increase with an ascent in the degree of personality instability.

Figure 3 shows that the brain activities in the left and right hippocampus under pleasant and human stimuli and the brain activities in right hippocampus under unpleasant stimuli significantly increased with an ascent in the degree of personality instability.

Figure 4 shows the brain activities in left and right thalamus under the human stimuli significantly increased with an ascent in the degree of personality instability.

\section{Discussion}

In today's society, stress on human relationships often causes people to suffer from mental disorders [2]. Our previous research showed that personality traits were crucial factors for determining whether people will have good relationships with their parents or not [7]. People who can handle human relationships without much stress are people who have a stable mental status.

The results in this study showed that the left and right hippocampus, amygdala, and thalamus of both the stable and unstable groups were significantly activated in pleasant, human, and unpleasant stimuli by ROI analysis. The brain activities in the left and right hippocampus under pleasant and human stimuli, the right hippocampus under unpleasant stimuli, and the left and right thalamus under human stimuli significantly increased with an ascent in the degree of personality instability using regression analysis.

There is extensive evidence that the amygdala is crucially involved in regulating stress effects on memory [4]. The amygdala has an important role in mediating initial responses to fearful stimuli. This is consistent with the 

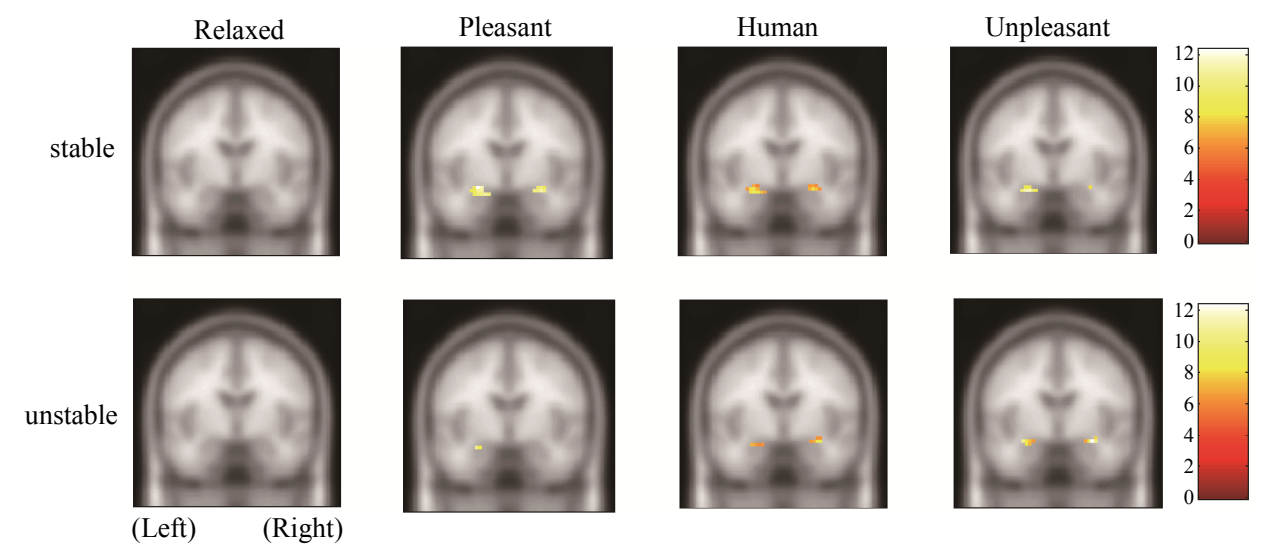

(a)
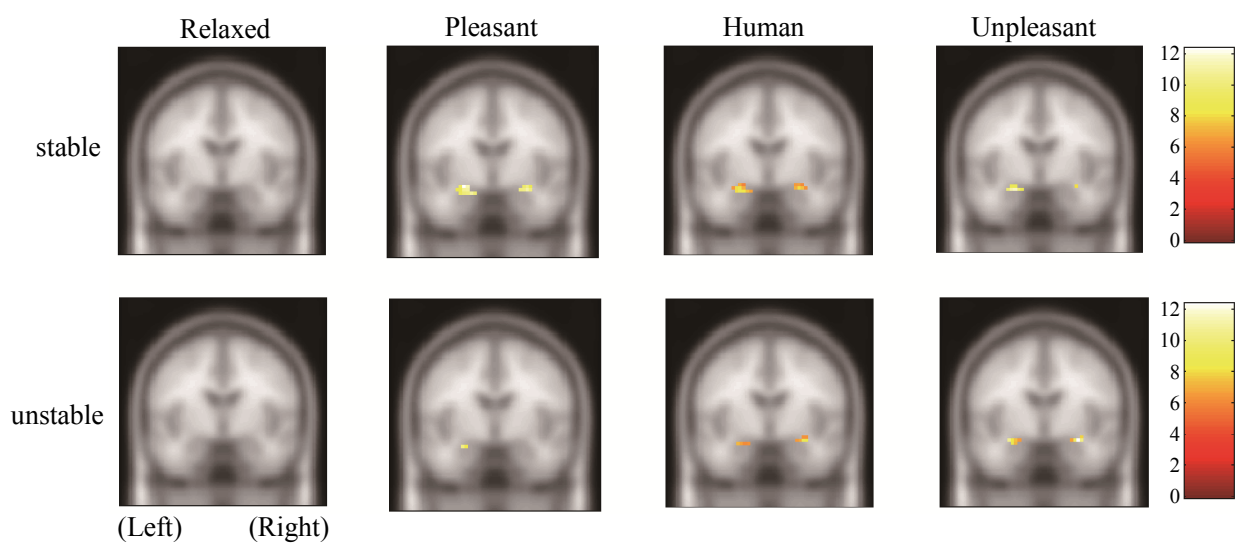

(b)
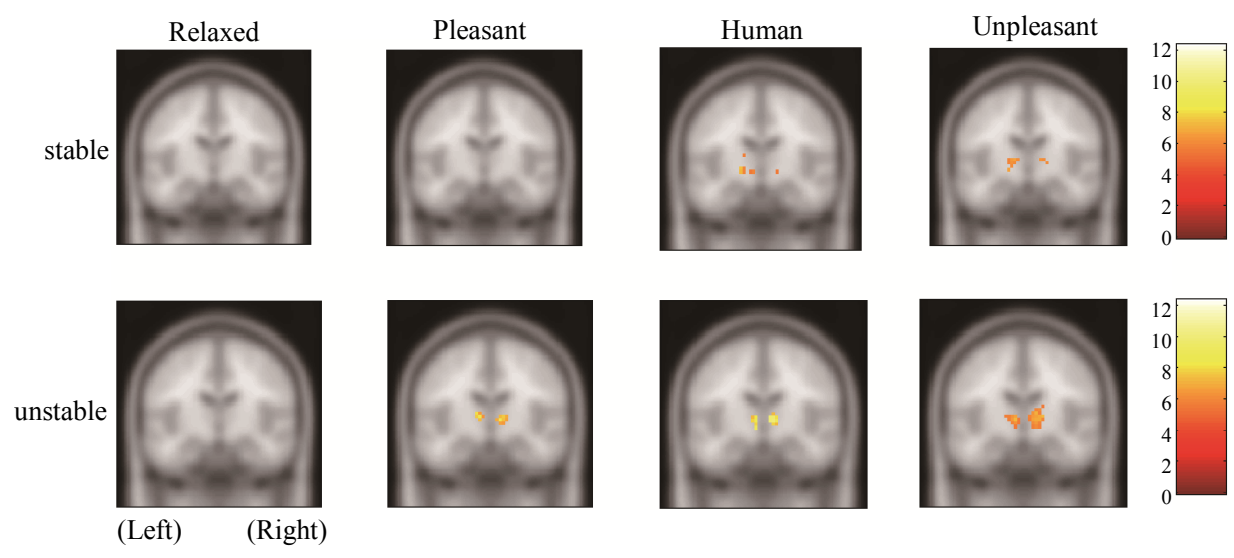

(c)

Figure 1. Functional activities of stable and unstable groups in relaxed, pleasant, human, and unpleasant stimuli. (a) Amygdala $[27,-6,-21]$; (b) Hippocampus [21, -7, -17]; (c) Thalamus [9, -12, 3].

demonstration of rapid amygdala habituation in humans exposed to fearful stimuli and is consistent with the concept that the primary role of the amygdala is to process novelty and ambiguity related to potentially threatening situations [8]. The amygdala has been reported to activate for both positively and negatively valenced emotional stimuli without different degrees between the two valences [9]. A study on drug addiction also showed that the amygdala regulates pleasant emotions [10]. Emotion enhances recollection-related activity in the hippocampus, whereas recollection enhances emotion-related activity in amygdala. The amygdala and hippocampus could be parts of a synergistic mechanism in which emotion enhances recollection and recollection enhances emotion [11].

The hippocampus is crucial for the formation of some 


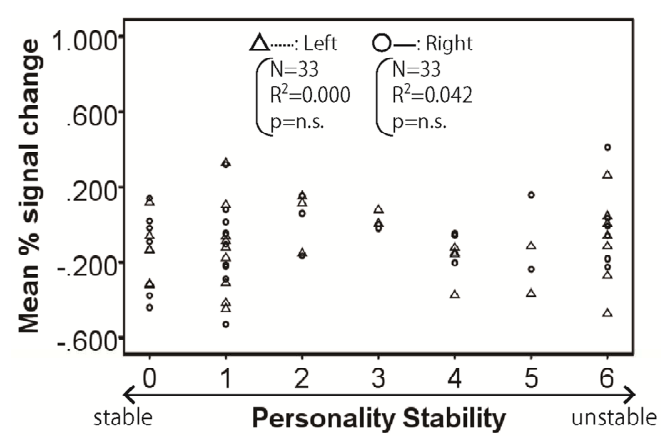

(a)

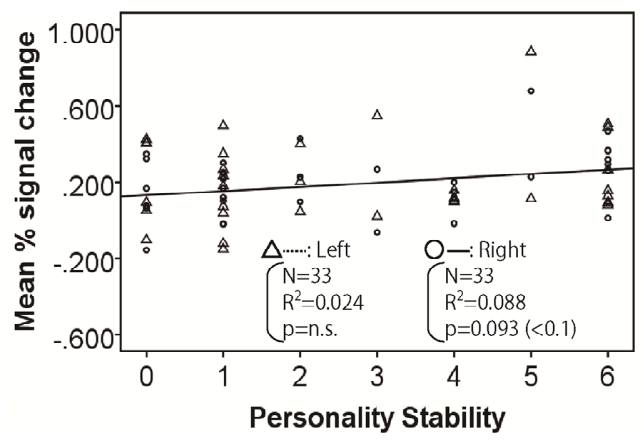

(c)

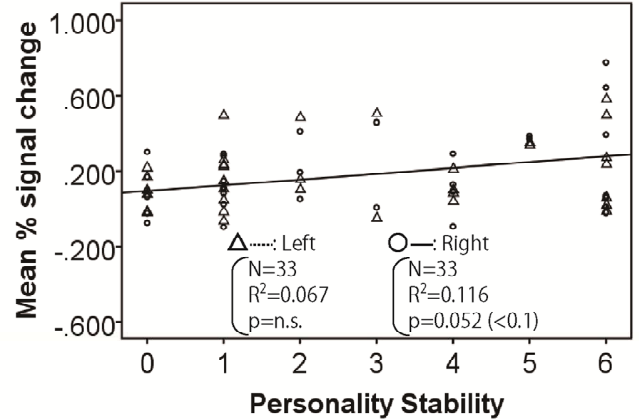

(b)

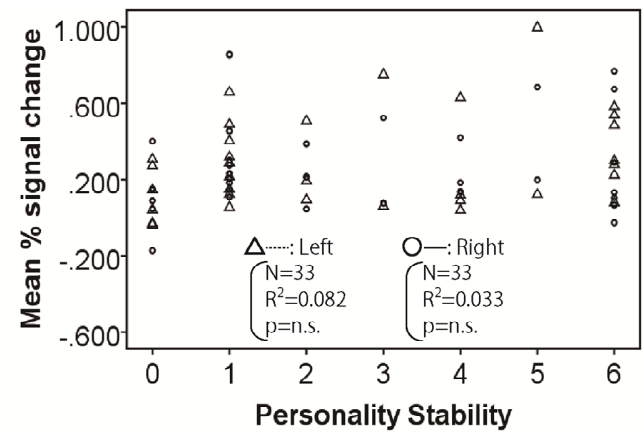

(d)

Figure 2. Relationship between mean \% signal change and personality stability in amygdala. The left activity is shown with a triangle and a dashed line, and the right activity is shown with a circle and a solid line. Only the significant and tended regression lines are shown in the figures. (a) Relaxed stimuli; (b) Pleasant stimuli; (c) Human stimuli; (d) Unpleasant stimuli.

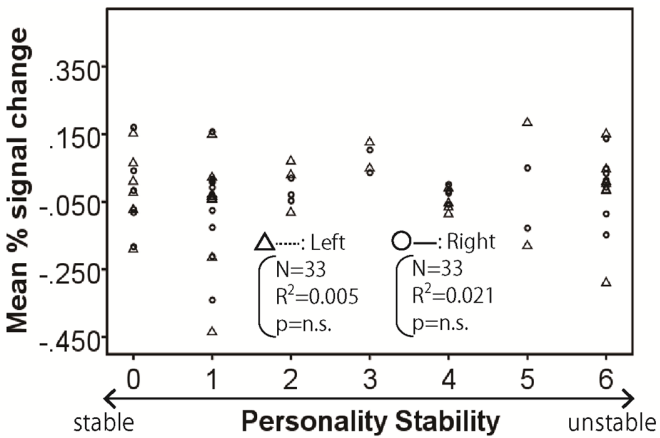

(a)

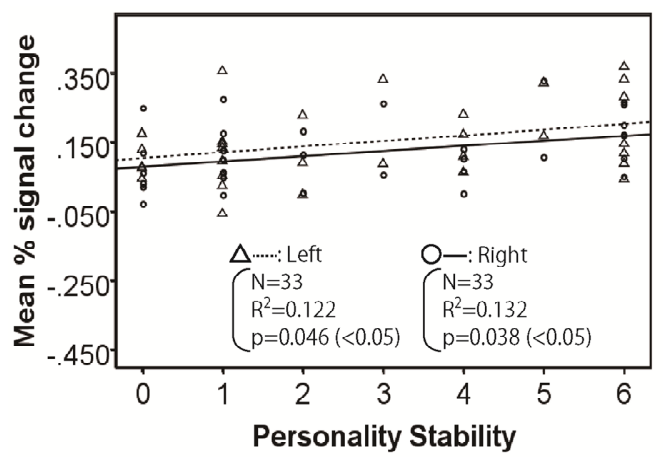

(c)

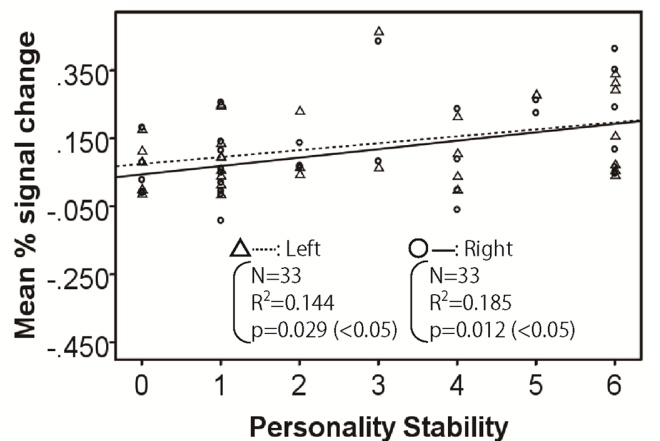

(b)

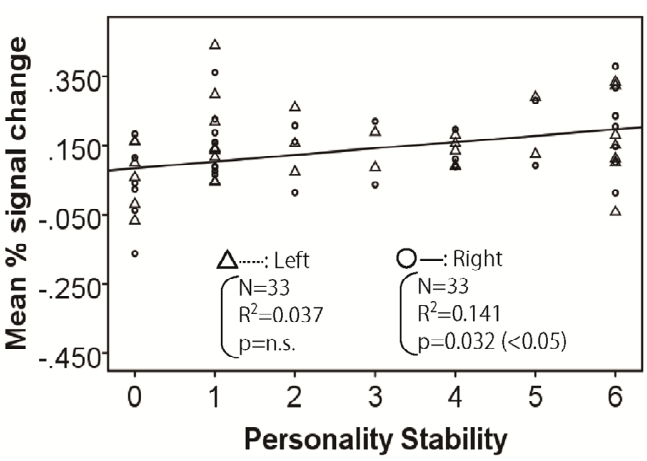

(d)

Figure 3. Relationship between mean \% signal change and personality stability in hippocampus. The left activity is shown with a triangle and a dashed line, and the right activity is shown with a circle and a solid line. Only the significant and tended regression lines are shown in the figures. (a) Relaxed stimuli; (b) Pleasant stimuli; (c) Human stimuli; (d) Unpleasant stimuli. 


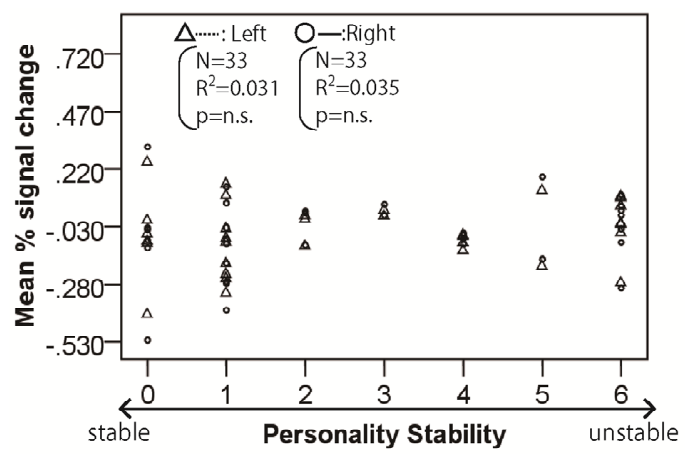

(a)

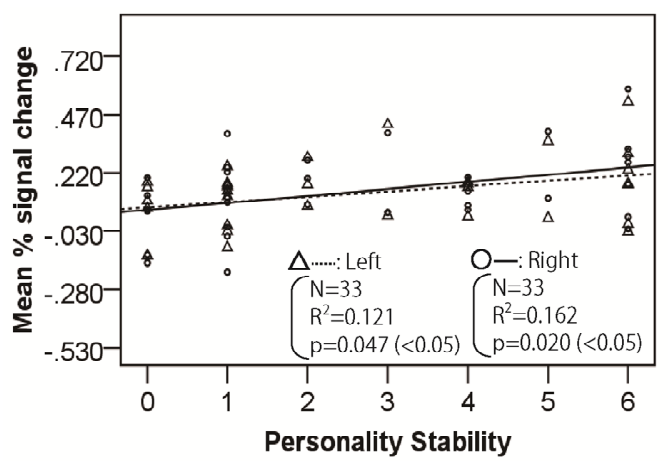

(c)

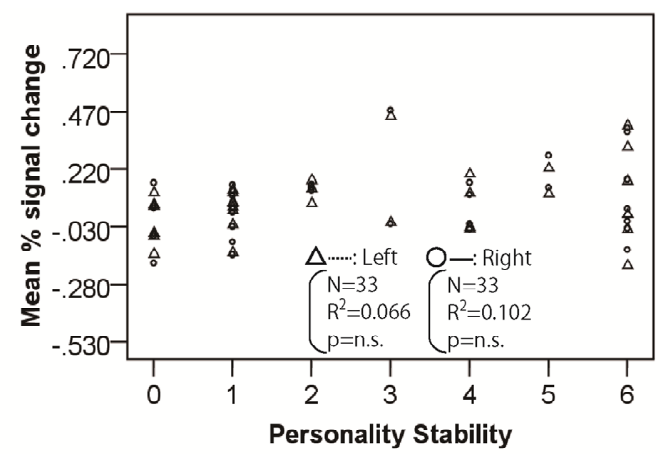

(b)

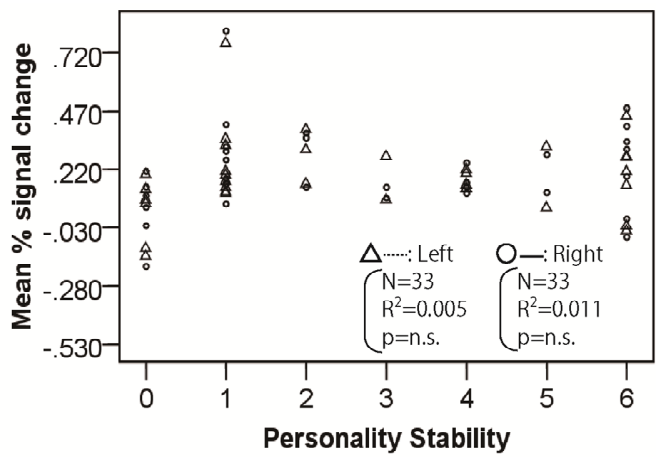

(d)

Figure 4. Relationship between mean \% signal change and personality stability in thalamus. The left activity is shown with a triangle and a dashed line, and the right activity is shown with a circle and a solid line. Only the significant and tended regression lines are shown in the figures. (a) Relaxed stimuli; (b) Pleasant stimuli; (c) Human stimuli; (d) Unpleasant stimuli.

types of declarative memories during the mnemonic process in humans [12]. The hippocampus-dependent periods for contextual fear memory are modulated by various treatments and conditions. In the other words, hippocampus dependency progressively decays over time, and there is a relationship between adult neurogenesis and the hippocampus-dependent period of associative fear memory. Dynamic interactions between hippocampal networks and the neocortex are necessary for supporting a role for CA1 in terms of intermediate or long-term retention, retrieval, and consolidation of spatial memory [13]. The acute stress causes stimulation of hippocampal outflow which evokes a remarkable and long-lasting inhibition of long-term potentiation in the frontal cortex [14]. This impairment in synaptic plasticity may be responsible for the acute deleterious effect of glucocorticoids on memory and for hippocampal atrophy in chronic situations. Thus, smaller hippocampal volume is associated with severe human-related stress early in life and combat-related posttraumatic stress disorder (PTSD) $[15,16]$.

The thalamus is implicated in threat processing in an inter-community communication in the brain. And the inhibitory thalamic reticular nucleus is a key node in the brain's circuit for attention [17]. The thalamus contains multiple sensory nuclei, and the thalamus actively regulates information transmission to the cortex by modulat- ing the response of neurons according to behavioral demands [18]. The thalamus is a central part of a fast subcortical sensory pathway by which low-level visual information can reach the amygdala and the cortex without conscious awareness. The neuron of the amygdala projects directly and indirectly to the hippocampus to help direct attention to emotional stimuli and expedite their access to the cortex $[4,19]$. Furthermore, the sites where projections from the amygdala predominate and innervate the thalamic neurons with large terminals proximally may control the rapid shift of affective stimuli that is important for survival. Valence was negatively associated with inter-subject correlations in the thalamus involved in emotional processing [20], and the left thalamus showed hyperactivity during both positive and negative emotional conditions in major depressive disorders [21]. The thalamic nuclei serve a variety of functions in the brain beyond relaying sensory information including the regulation of arousal, the initiation and coordination of motor behavior, and the participation in high-level cognitive functions such as learning [22].

Our results showed the process of emotional stimuli in subcortices. First, all kinds of emotional stimuli could be assessed whether the stimuli were pleasant or unpleasant in the amygdala of both stable and unstable people. Then, this information could propagate to the hippocampus and 
thalamus. In the hippocampus, the input is judged referring to the memory, and this process increases with an ascent in the degree of personality instability, regardless of types of emotional stimuli. In the thalamus, the response to threatening, fearful, and unpleasant input related to human relationships increases with an ascent in the degree of personality instability. This thalamic excessive response in addition to the hippocampal reaction could consolidate the unpleasant memory in the brain. Given the fact that the top cause of people's stress is human-related problems, the overreaction to the humanrelated stimuli in the hippocampus and thalamus could cause stress-related mental disorders such as PTSD and neurosis.

Our results suggest that mentally unstable people could have a hippocampus and thalamus sensitive to an unpleasant human-related event. And the overreaction of hippocampus and thalamus to a human-related problem as well as an unpleasant stimulus could make people store a strong memory of threat on the event. In other words, when mentally unstable people have personality problems in human relationships at work and in their families, insufficient processes related to human relationships would show in the hippocampus and thalamus, and this could cause mental and psychosomatic symptoms.

\section{Acknowledgements}

This work is partially supported by a Grant-in-Aid from the Ministry of Education, Culture, Sports, Science and Technology, JP (23500527).

This research is also supported by NICT, Japan.

The authors would like to thank Dr. Yasuto Tanaka and Dr. Testuto Minami for their valuable comments and assistance.

\section{REFERENCES}

[1] Ministry of Health, Labour and Welfare, "Mental Health," 2012.

http://www.mhlw.go.jp/kokoro/speciality/detail_depressi ve.html

[2] Ministry of Health, Labour and Welfare, "Comprehensive Survey of Living Conditions," 2010. http://www.mhlw.go.jp/toukei/list/20-21.html

[3] Ministry of Labour and Welfare, "Survey of State of Employees' Health," 2007. http://www.mhlw.go.jp/toukei/list/49-19.html

[4] B. Roozendaal, B. S. McEwen and S. Chattarji, "Stress, Memory and the Amygdala," Nature Reviews Neuroscience, Vol. 10, No. 6, 2009, pp. 423-433. http://dx.doi.org/10.1038/nrn2651

[5] I. Ueno, "Psychological Assessment Handbook," Nishimura-Shyoten, Tokyo, 2001.

[6] CMI, "A Brief History of the Cornell Medical Index (CMI) in Weill Cornell Medical Library,” 2012.
http://library.weill.cornell.edu/About/cornellmedindex.html

[7] Y. Mizuno-Matsumoto, T. Hayashi, E. Okamoto, T. Asakawa, K. Sawamura, R. Ishii, S. Ukai and K. Shinosaki, "Measurement of Personality Stability in Infants and Young Adults under Emotional Stimuli Using a Brain Functional Reaction Method," International Journal of Intelligent Computing in Medical Sciences and Image Processing (IC-MED Journal), Vol. 4, No. 1-2, 2011, pp. 39-64.

[8] N. H. Kalin, S. E. Shelton, R. J. Davidson and A. E. Kelley, "The Primate Amygdala Mediates Acute Fear but Not the Behavioral and Physiological Components of Anxious Temperament," The Journal of Neuroscience, Vol. 21, No. 6, 2001, pp. 2067-2074.

[9] H. Garavan, J. C. Pendergrass, T. J. Ross, E. A. Stein and R. Risinger, "Amygdala Response to both Positively and Negatively Valenced Stimuli," NeuroReport, Vol. 12, No. 12, 2001, pp. 1-5. http://dx.doi.org/10.1097/00001756-200108280-00036

[10] J. A. Kauer and R. C. Malenka, "Synaptic Plasticity and Addiction," Nature Reviews Neuroscience, Vol. 8, No. 11, 2007, pp. 844-858. http://dx.doi.org/10.1038/nrn2234

[11] F. Dolcos, K. S. LaBar and R. Cabeza, "Remembering One Year Later: Role of the Amygdala and the Medial Temporal Lobe Memory System in Retrieving Emotional Memories," Proceedings of the National Academy of Sciences, Vol. 102, No. 7, 2005, pp. 2626-2631. http://dx.doi.org/10.1073/pnas.0409848102

[12] T. Kitamura, Y. Saitoh, N. Takashima, A. Murayama, Y. Niibori, H. Ageta, M. Sekiguchi, M. H. Sugiyama and K. Inokuchi, "Adult Neurogenesis Modulates the Hippocampus-Dependent Period of Associative Fear Memory," Cell, Vol. 139, No. 4, 2009, pp. 814-827.

http://dx.doi.org/10.1016/j.cell.2009.10.020

[13] D. R. Vago, A. Bevan and R. P. Kesner, "The Role of the Direct Perforant Path Input to the CA1 Subregion of the Dorsal Hippocampus in Memory Retention and Retrieval," Hippocampus, Vol. 17, No. 11, 2007, pp. 977-987. http://dx.doi.org/10.1002/hipo.20329

[14] C. Rocher, M. Spedding, C. Munoz and T. M. Jay, "Acute Stress-Induced Changes in Hippocampal/Prefrontal Circuits in Rats: Effects of Antidepressants," Cerebral Cortex, Vol. 14, No. 2, 2004, pp. 224-229. http://dx.doi.org/10.1093/cercor/bhg122

[15] J. D. Bremner, P. Randall, T. M. Scott, R. A. Bronen, J. P. Seibyl, S. M. Southwick, R. C. Delaney, G. McCarthy, D. S. Charney and R. B. Innis, "MRI-Based Measurement of Hippocampal Volume in Patients with Comat-Related Posttraumatic Stress Disorder," Journal of the American Psychiatric Association, Vol. 152, No. 7, 1995, pp. 973981.

[16] M. Vythilingam, C. Heim, J. Newport, A. H. Miller, E. Anderson, R. Bronen, M. Brummer, L. Staib, E. Vermetten, D. S. Charney, C. B. Nemeroff and J. D. Bremner, "Childhood Trauma Associated with Smaller Hippocampal Volume in Women with Major Depression," Journal of the American Psychiatric Association, Vol. 159, No. 12, 2002, pp. 2072-2080.

http://dx.doi.org/10.1176/appi.ajp.159.12.2072 
[17] J. Kinnison, S. Padmala, J. M. Choi and L. Pessoa, "Network Analysis Reveals Increased Integration during Emotional and Motivational Processing," The Journal of Neuroscience, Vol. 32, No. 24, 2012, pp. 8361-8372. http://dx.doi.org/10.1523/JNEUROSCI.0821-12.2012

[18] Y. Nakagawa and T. Shimogori, "Diversity of Thalamic Progenitor Cells and Postmitotic Neurons," European Journal of Neuroscience, Vol. 35, No. 10, 2012, pp. 15541562. http://dx.doi.org/10.1111/j.1460-9568.2012.08089.x

[19] B. Zikopoulos and H. Barbas, "Pathways for Emotions and Attention Converge on the Thalamic Reticular Nucleus in Primates," The Journal of Neuroscience, Vol. 32, No. 15,2012 , pp. $5338-5350$. http://dx.doi.org/10.1523/JNEUROSCI.4793-11.2012

[20] L. Nummenmaa, E. Glerean, M. Viinikainen, I. P. Jaakelainen, R. Hari and M. Sams, "Emotion Promote Social
Interaction by Synchronizing Brain Activity across Individuals," Proceedings of the National Academy of Sciences, Vol. 109, No. 24, 2012, pp. 9599-9604. http://dx.doi.org/10.1073/pnas.1206095109

[21] C. Diener, C. Kuehner, W. Brusniak, B. Ubl, M. Wessa and H. Flor, "A Meta-Analysis of Neurofunctional Imaging Studies of Emotion and Cognition in Major Depression," NeuroImage, Vol. 61, No. 3, 2012, pp. 677-685. http://dx.doi.org/10.1016/j.neuroimage.2012.04.005

[22] T. Suslow, H. Kugel, H. Reber, J. Bauer, U. Dannlowski, A. Kersting, V. Arolt, W. Heindel, P. Ohrmann and B. Egloff, "Automatic Brain Response to Facial Emotion as a Function of Implicitly and Explicitly Measured Extraversion," NeuroScience, Vol. 167, No. 1, 2010, pp. 111123. http://dx.doi.org/10.1016/j.neuroscience.2010.01.038 